\title{
Das Programm der Nationalsozialistischen Deutschen Arbeiterpartei
}

1. Wir fordern den Zusammenschluß aller Deutschen auf Grund des Selbstbestimmungsrechtes der Völker zu einem Groß-Deutschland.

2. Wirfordern die Gleichberechtigung des Deutschen Volkes gegenüber den anderen Nationen, Aufhebung der Friedensverträge von Versailles und St.Germain.

3.W ir fordern Land und Boden (Kolonien) zur Ernährung unseres Volkes und Ansiedlung unseres Bevölkerungs-Überschusses.

4S taatsbürger kannnursein, wer Volksgenosseist. Volksgenossekann nur sein, wer Deutschen Blutes ist, ohne Rücksicht auf Konfession. Kein Jude kann daher Volksgenosse sein.

5.Wer nicht Staaisbürger ist, soll nur als Gast in Deutschland leben können und muß unter Fremdengesetzgebung stehen.

6. Das Recht, über Führung und Gesetze des Staates zu bestimmen, darf nur dem Staatsbürger zustehen. Daher fordern wir, daß jedes öfentliche Amt, gleichgültig welcher Art, gleich ob im Reich, Land oder Gemeinde, nur durch Staatsbürger bekleidet werden darf.

Wir bekämpfen diekorrumpierende Parlamentswirtschafteiner Stellenbesetzung nur nach Parteigesichtspunkten ohne Rücksichten auf Charakter und Fähigkeiten.

7.W ir fordern, daß sich der Staat verpflichtet, in erster Linie für die Erwerbs- und Lebensmöglichkeit der Staatsbürger zu sorgen. Wenn es nicht möglich ist, die Gesamtbevölkerung des Staates zu ernähren, so sind die Angehörigen fremder Nationen (Nicht-Staatsbürger) aus dem Reiche auszuweisen.

8. J ede weitere Einwanderung Nicht-Deutscher ist zu verhindern. Wir fordern, daß alle Nicht-Deutschen, die seit dem 2. August 1914 in Deutschland eingewandert sind, sofort zum Verlassen des Reiches gezwungen werden.

9.Alle Staatsbürger müssen gleiche Rechte und Pflichten besitzen.

10.E rste Pflicht jedes Staatsbürgers muß sein, geistig oder körperlich zu schaffen. Die Tätigkeit des Einzelnen darf nicht gegen die Interessen der Allgemeinheit verstoßen, sondern muß im Rahmen des Giesamten und zum Nutzen Aller erfolgen. Daher fordern wir:

11.A bschaffung des arbeits- und mühelosen Einkommens. Brechung der Zinsknechtschaft.

12.I $m$ Hinblick auf die ungeheuren Opfer an Gut und Blut, die jeder Krieg vom Volke fordert, muß die persönliche Bereicherung durch den Krieg als Verbrechen am Volke bezeichnet werden. Wir fordern daher restlose Einziehung aller Kriegsgewinne.

13. Wir fordern die Verstaatlichung aller (bisher) bereits vergesellschafteten (Trusts) Betriebe.

14.W ir fordern Gewinnbeteiligung an Großbetrieben.

15.Wir fordern einen großzügigen Ausbau der Altersversorgung.

16.W ir fordern die Schaffung eines gesunden Mittelstandes und seine Erhaltung, sofortigeKommunalisierung derGroß-Warenhäuser undihre Vermietung zu billigen Preisen an kleine Gewerbetreibende, schärfste Berücksichkigung aller kleinen Gewerbetreibenden bei Lieferung an den Staat, die Länder oder Gemeinden.

17. Wir fordern eine unseren nationalen Bedürfnissen angepaßte Bodenreform, Schaffung eines Gesetzes zur unentgeltlichen Enteignung von Boden für gemeinnützige Zwecke. Abschaffung des Bodenzinses und Verhinderung jeder Bodenspekulation.
18. Wir fordern den rücksichtslosen Kampf gegen diejenigen, die durch ihre Tätigkeit das Gemeininteresse schädigen. Gemeine Volksverbrecher, Wucherer, Schieber usw. sind mit dem Tode zu bestrafen, ohne Rücksichtnahme auf Konfession und Rasse.

19. W ir fordern Ersatz für das der materialistischen Weltordnung dienende römische Recht durch ein Deutsches Gemeinrecht.

20IJm jedem fähigen und fleißigen Deutschen das Erreichen höherer Bildung und damit das Einrücken in führende Stellung zu ermöglichen, hat der Staat für einen gründlichen Ausbau unseres gesamten Volksbildungswesens Sorge zu tragen.

Die Lehrpläne aller Bildungsanstalten sind den Erfordernissen des praktischen Lebens anzupassen. Das Erfassen des Staatsgedankens muß bereits mit dem Beginn des Verständnisses durch die Schule (Staatsbürgerkunde) erzielt werden. Wir fordern die Ausbildung besonders veranlagter Kinder armer Eltern ohneRücksicht auf deren Stand oder Beruf ouf Staatskosten.

21.D er Staat hat für die Hebung der Volksgesundheit zu sorgen durch den Schutz der Mutter und des Kindes, durch Verbot der Jugendarbeit, durch Herbeiführung der körperlichen Ertüchtigung mittels gesetzlicher Festlegung einer Turn-und Sportpflicht, durch gröBteUnterstützung aller sich mit körperlicher Jugendausbildung beschäftigenden Vereine.

22. Wir fordern die Abschaffung der Söldnertruppe und die Bildung eines Volksheeres.

23. W ir fordern den gesetzlichen Kampf gegen die bewußte politische Lüge und ihre Verbreitung durch die Presse. Um die Schaffung einer Deutschen Presse zu ermöglichen, fordern wir, daß: a) sämtliche Schriftleiter und Mitarbeitervon Zeitungen, die in deutscher Spracheerscheinen, Volksgenossen sein müssen, b) nichtdeutsche Zeitungen zu ihrem Erscheinen der ausdrücklichen Gienehmigung des Staates bedürfen. Sie dürfen nichtin deutscher Sprache gedrucktwerden, c) jede finanzielle Beteiligung an Deutschen Zeitungen oder deren Beeinflussung durch NichtDeutsche gesetzlich verboten wird und fordern als Strafe für Ubertretungen die Schließung eines solchen Zeitungsbetriebes sowie die sofortige Ausweisung der daran beteiligten Nicht-Deutschen aus dem Reich. Zeitungen, die gegen das Gemeinwohl verstoßen, sind zu verbieten. Wir fordern den gesetzlichen Kampf gegen eine Kunst-und Literatur-Richtung, die einen zersetzenden Einfluß auf unser Volksleben ausübt und dieSchließung von Veranstaltungen, die gegen vorstehende Forderungen verstoßen.

24.W ir fordern die Freiheit aller religiösen Bekenntnisseim Staat, soweit sie nicht dessen Bestand gefährden oder gegen das Sittlichkeits- und Moralgefühl der germanischen Rasse verstoßen.

Die Partei als solche vertritt den Standpunkt eines positiven Christentums, ohne sich konfessionell an ein bestimmtes Bekenntnis zu binden. Sie bekämpft den jüdisch-materialistischen Geist in und außer uns und istüberzeugt, daßeine dauernde Genesung unseres $V$ olkes nur erfolgen kann von innen heraus auf der Grundlage: Gemeinnutz vor Eigennutz.

25.Zur Durchführung alles dessen fordern wir: Die Schaffung einer starken Zentralgewalt des Reiches. Unbedingte Autorität des politischen Zentralparlaments über das gesamte Reich und seine Organisationen imallgemeinen.DieBildungvonStände-undBerufskammernzurDurchführung der vom Reich erlassenen Rahmengesetze in den einzelnen Bundesstacten. DieFührer der Partei versprechen, wenn nötig unter Einsatz des eigenen Lebens für die Durchführung der vorstehenden Punkte rücksichtslos einzutreten. 


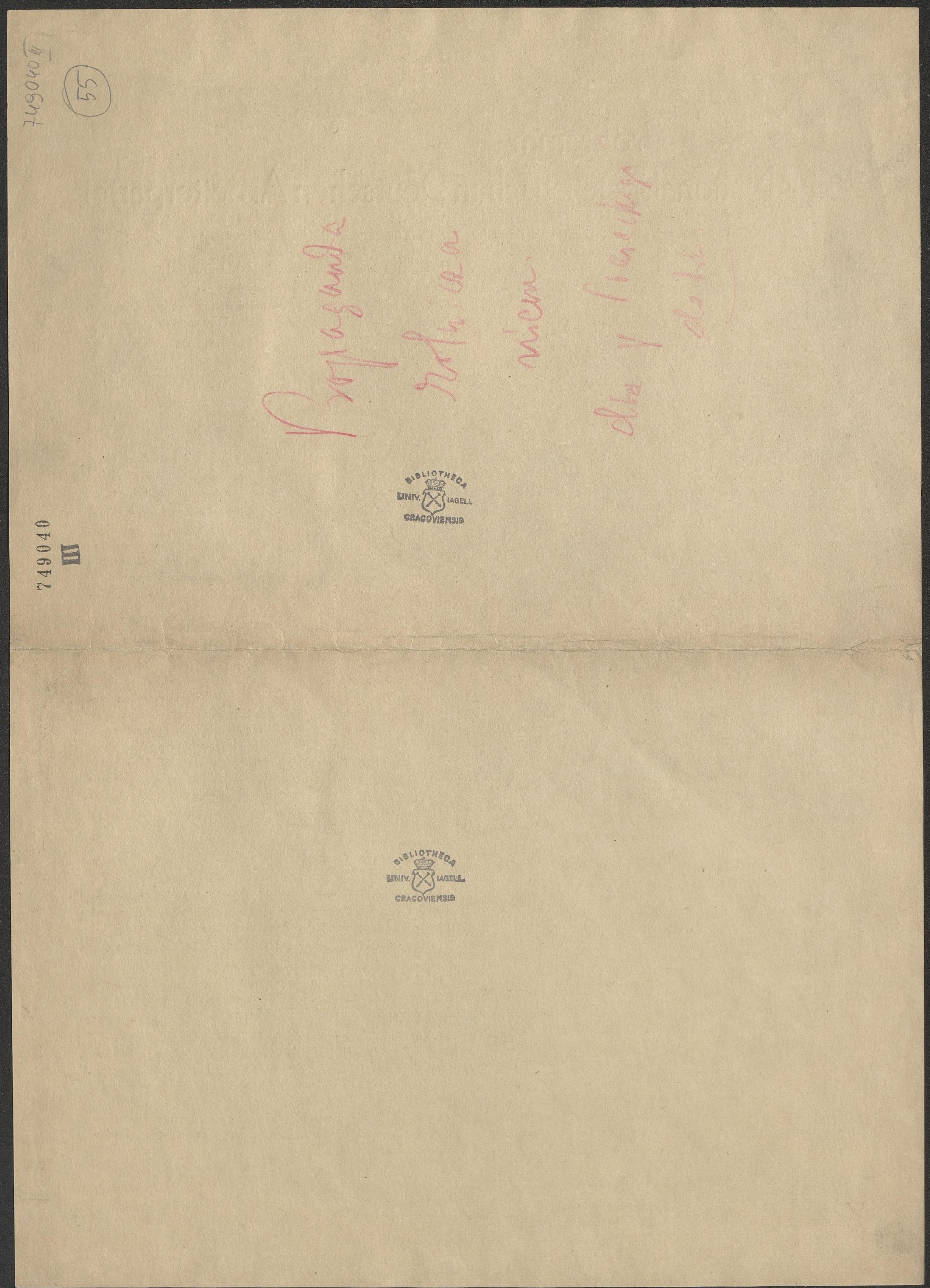

